\title{
A Sharing and Delivery Scheme for Monitoring TMO-Based Real-Time Systems*
}

\author{
Yoon-Seok Jeong, Tae-Wan Kim**, and Chun-Hyon Chang \\ Konkuk University, \\ Seoul 143-701, Korea \\ \{ysjeong, twkim, chchang\}@konkuk.ac.kr
}

\begin{abstract}
Devices and systems used in distributed environments, such as on trains or in power plants, should be able to respond to external changes in realtime. Real-time middleware such as one based on the TMO (Time-Triggered Message-Triggered Object) model is considered recently as a choice to adapt real-time properties to distributed systems. TMO middleware guarantees that systems in distributed environments operate in a reliable manner. This middleware does not have an adequate monitoring infrastructure or supporting tools used in distributed environments, however. As such, this paper proposes TSDS (TMO-based Sharing and Delivery Scheme) as a part of the monitoring infrastructure. This is configured within the TMO middleware to share and deliver monitoring data among TMO-based real-time systems. The results of experiments show that the TSDS instrumentation overhead is less than $1 \mathrm{~ms}$. This means that TSDS has little effect on the operation of the middleware.
\end{abstract}

\section{Introduction}

A real-time system aims to ensure a specific service is provided within a given period of time [1]. The performance of a real-time system depends on how accurately timing constraints are met through the use of real-time monitoring. Monitoring is essential for maintaining real-time systems in a stable manner, and many monitoring tools are being developed to support real-time systems. As well, tools that monitor the TMO (Time-Triggered Message-Triggered Object)-based real-time systems (hereinafter referred to as "TMO system") were developed [2, 8, 9]. However, existing tools that focus on single system-based monitoring cannot enable the sharing and delivery of data among systems, which is required to monitor distributed TMO systems. In addition, all or part of the monitoring function is executed at the application layer, requiring developer intervention, and eventually causing difficulty in automating monitoring.

In order to address such problems, this paper presents a TMO-based Sharing and Delivery Scheme (TSDS), which is a scheme for sharing and delivering monitoring data among TMO systems. This configures an infrastructure for monitoring within the

This research was supported by the MIC (Ministry of Information and Communication), Korea, under the ITRC (Information Technology Research Center) support program supervised by the IITA (Institute of Information Technology Assessment).

** Corresponding author. 
TMO middleware, an essential part of the TMO system, thereby allowing for the sharing and delivery of monitoring data.

The paper is structured as follows. Chapter 2 describes the TMO model and the problems in existing monitoring architectures. Chapter 3 provides a detailed description of the proposed TSDS. The results of the experiments on the TSDS instrumentation overhead are shown in Chapter 4. Finally, chapter 5 presents the direction for future research.

\section{Backgrounds}

\subsection{TMO Model}

The proposed scheme in this paper is modeled in such a way as to utilize the proven functions provided by the TMO model. The TMO structuring scheme was established in the early 1990's with a concrete syntactic structure and execution semantics for economical and reliable design along with an implementation of realtime systems [6, 7]. TMO is a syntactically minor and semantically powerful extension of the conventional object(s). As depicted in Fig. 1, the basic TMO structure consists of four parts.

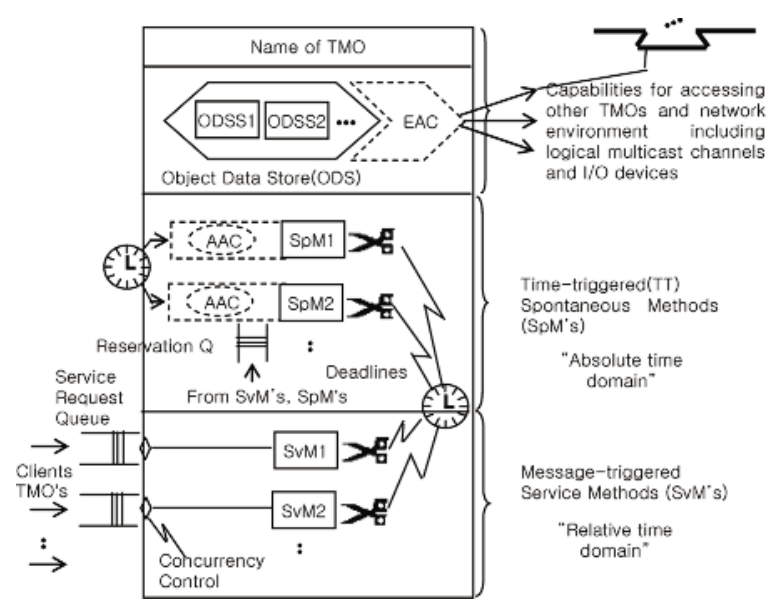

Fig. 1. The Basic Structure of TMO

- Spontaneous Method (SpM): A new type of method. A SpM is triggered when the real-time clock reaches specific values determined at design time. The SpM has an AAC (Autonomous Activation Condition), which is a specification of the timewindows for execution of the SpM.

- Service Method $(S v M)$ : A conventional service method. A SvM is triggered by service request messages from clients.

- Object Data Store (ODS): The basic units of storage which can be exclusively accessed by a certain TMO method at any given time or shared among TMO methods (SpMs or SvMs).

- Environment Access Capability (EAC): The list of entry points to remote object methods, logical communication channels, and I/O device interfaces. 


\subsection{Existing Monitoring Architecture}

Some studies related to monitoring TMO systems have used architecture at the application layer to implement monitoring functions as shown in Fig. 1(a) $[1,8,9]$. A monitoring sensor positioned within a TMO application, an essential part of the monitor, gathers data on the corresponding object in the TMO application, and keeps them in a data store. This architecture has no direct effect on the operation of the middleware because monitoring TMO systems is conducted at the application layer. The drawback of this architecture is that a developer has to make monitoring elements such as a data store, sensors and a communication link for the sharing and delivery of monitoring data.

The other architecture used to implement monitoring functions is depicted in Fig. 2(b) [2]. A dedicated monitoring thread is added in the middleware and called up according to the middleware scheduling. While this architecture reduces developer intervention to make a store data and a sensor, the data stored in the data store can be accessed only by TMO objects in a single TMO system because the data store in this architecture is dependent on the middleware.

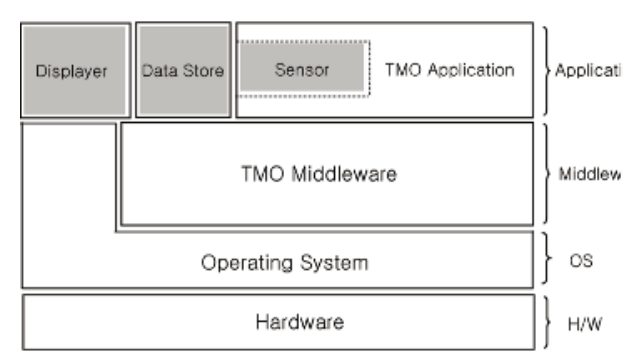

(a) at the Application Layer

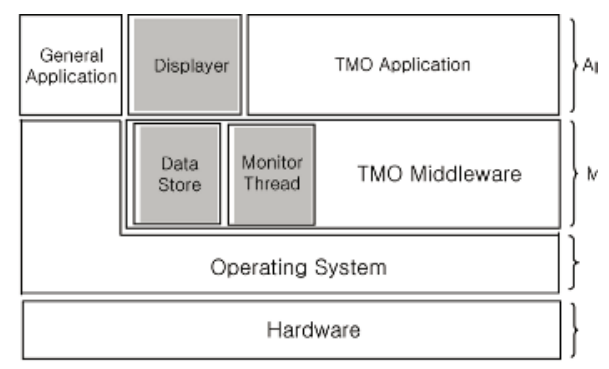

(b) at the Middleware Layer

Fig. 2. The Monitoring Architecture by Layer

In summary, the existing architectures for monitoring TMO systems do not have supported the sharing and delivery of monitoring data in distributed environments. Given that the TMO middleware supports distributed environments, it is difficult for both monitoring architectures, which are considering a stand-alone system environment, to be used for monitoring several TMO systems in distributed environments. Thus, a structure which supports data sharing and data delivery in distributed environments should be designed for monitoring TMO systems.

\section{TMO-Based Sharing and Delivery Scheme (TSDS)}

\subsection{Revised TMO Model for Monitoring}

The various types of middleware to realize the TMO model have been implemented in distributed environments $[3,4,6,10]$. Each middleware has referred to the TMO model in Fig. 1 as a functional architecture. The TMO model does not include essential functions in distributed environments such as real-time monitoring, 
dynamic analysis, and fault-tolerance, however. As such, this paper has considered a monitoring concept from the outset of modeling in order to allow monitoring functions to be included as an infrastructure within the TMO-based middleware. Fig. 3 shows the proposed TMO model that supports monitoring functions. Some elements are added to this model in order for the monitoring concept to be applied to the TMO model.

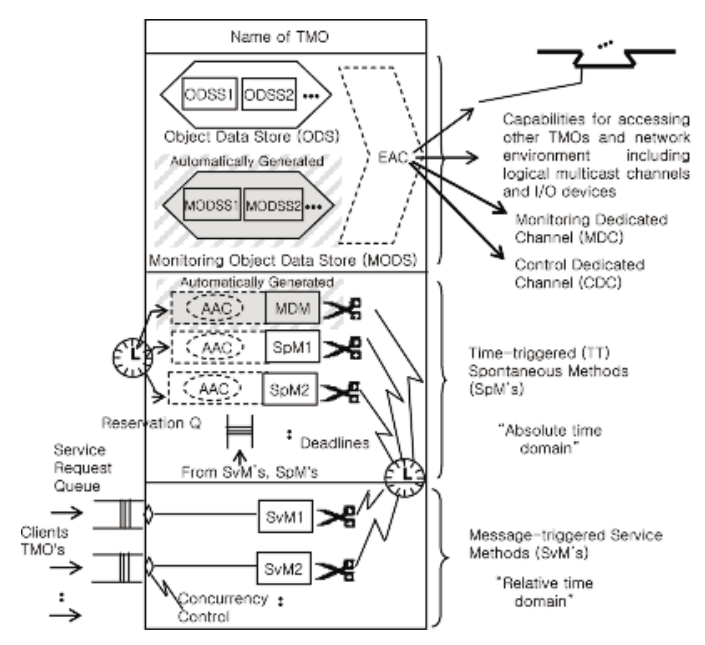

Fig. 3. The Structure of the TMO Model that Supports Monitoring Functions

- Monitoring ODS (MODS): Refers to the storage for sharing the results of monitoring TMO objects. It is the extended type of ODS that is assigned to each TMO object by a unit of MODS Segment (MODSS).

- Monitoring Dedicated Method (MDM): Refers to the dedicated SpM method for monitoring that is activated periodically in the same manner as general SpMs and transfers monitoring data in the data store.

- Monitoring Dedicated Channel (MDC): Refers to the dedicated channel used for the transfer of monitoring data among TMO systems.

- Control Dedicated Channel (CDC): Refers to the dedicated channel used for receiving control and feedback data from external systems or applications.

Our focus in this paper is the MODS, MDC, and CDC among abovementioned elements and the detailed elements in the middleware related to sharing and delivering the monitoring data. These elements are based on Linux TMO System (LTMOS) out of various types of the middleware as the basic platform for designing and implementing the sharing and delivery scheme. For more details of LTMOS, refer to $[4,10]$.

\subsection{Monitoring Object Data Store (MODS)}

The existing ODS was proposed as a storage to share data among TMO objects [6, 7]. On the other hand, the proposed MODS is designed to store monitoring data. 
Fig. 4 shows the monitoring architecture proposed in this paper. Compared with ODS, MODS as a data store is positioned between the middleware and the application layer. This means that all the work related to defining and generating MODS is managed by the middleware. Thus, developers do not have to do MODS related work. This is caused by the type of data stored in the data stores. MODS keeps the formalized data such as execution time while ODS stores the various types of data that developers need. Therefore, it doesn't need additional information from the developers to define MODS.

MODS can support data sharing because it is designed to face the application layer. MODS is the hybrid of the two data stores used in the legacy monitoring schemes. In other words, MODS is designed in such a way as to be defined and created by the middleware, thereby preventing developer intervention and supporting automated monitoring. The data stored in MODS can be accessed by all the applications because it is designed to be independent of the middleware. Therefore, MODS is a data store that can overcome the drawbacks of the existing data stores and is suitable for distributed real-time monitoring.

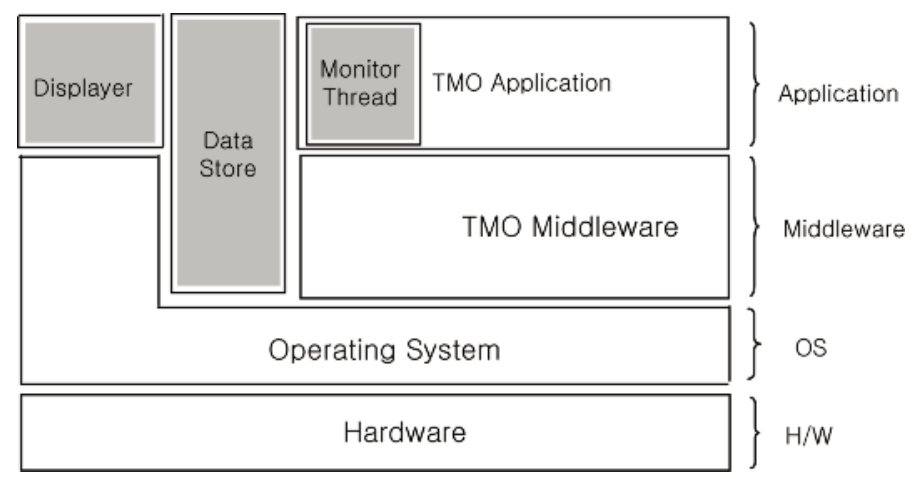

Fig. 4. The Revised Monitoring Architecture

\subsection{Delivery Structure for Monitoring Data}

Existing monitoring tools do not provide a special way to transfer monitoring data among TMO systems. Given that TMO systems operate in distributed environments, tools should be able to basically monitor each TMO system and deliver the gathered data to the server for analysis or other systems. As such, this paper presents a TMObased delivery structure, which is a structure for delivery of monitoring data among TMO systems.

This structure is designed to have two separate layers that make functions of the data manipulation and data delivery independent of each other. This functional independency allows that a function for data manipulation can be used in all the TMO systems with different communication environments. The layer for data manipulation contains functions related to gathering, storing, and analyzing monitoring data. EMCB (Extended Method Control Block) as an internal data store, sensors which 
gather monitoring data, and MODS, which supports data sharing, are monitoring elements for data manipulation. This paper focuses only on MODS out of these monitoring elements. The layer for data delivery consists of logical and physical links that support data delivery among TMO systems. TCP/IP or Serial for physical data delivery will be selected depending on the implemented communication environments. Also, a general channel or RMMC (Real-time Multicast Memory replication Channel) as a logical link, which establishes connection among TMO systems and manages communication sessions, can be used [5].

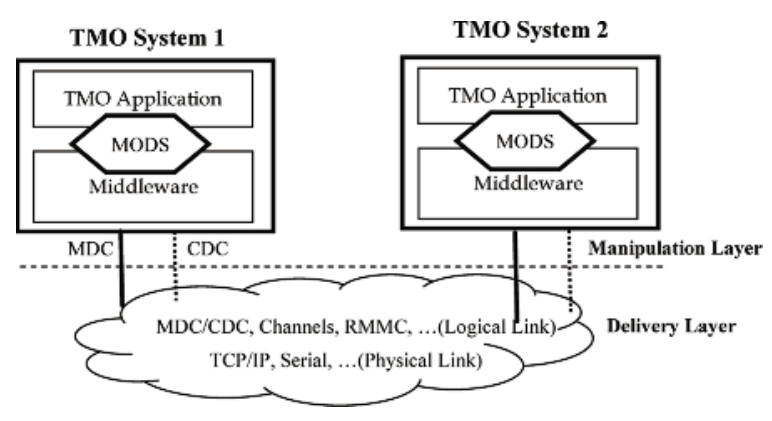

Fig. 5. A Delivery Structure for Monitoring Data

In this paper, the MDC and CDC are used as a logical link. The TMO model basically provides a logical communication link--channel--for connection among methods. Each channel is assigned a channel number, and data can be transferred via a channel that is opened with the same number. The MDC and CDC presented in this paper are designed based on such TMO model channels. Unlike RMMC and a general channel, the MDC and CDC are generated and managed by the middleware, which prevents the need for developer intervention for the delivery of monitoring data and allows automating monitoring.

(a) Monitoring Dedicated Channel (MDC): Refers to the dedicated channel for delivery of monitoring data. The middleware delivers monitoring data stored in MODS to other systems through MDC. To prevent collision with common channels, the MDC uses a specific reserved channel number.

(b) Control Dedicated Channel $(C D C)$ : Refers to the feedback channel that is designed to receive control and feedback information from external systems. Like MDC, it uses a specific reserved channel number.

\section{Experiments}

\subsection{Purpose and Method}

For the purpose of our experiment, the legacy middleware (hereinafter referred to as "Pure Middleware") and the middleware in which TSDS is applied (hereinafter referred to as "Monitoring Middleware") are experimented with under the same 
condition. Basically, the Monitoring Middleware is configured to share monitoring data with the other system in a periodic manner. Then, actual activation intervals were measured for OMMT (Outgoing Message Management Thread) and IMMT (Incoming Message Management Thread) which are middleware threads related to the sharing and delivery of monitoring data and should be activated at every $10 \mathrm{~ms}$. By conducting a comprehensive comparison between these intervals by the thread, the magnitude of loads generated by monitoring using TSDS was identified.

\subsection{Experimental Results}

Figs. 6(a) and 6(b) show the activation intervals of the IMMT thread in the Pure Middleware and Monitoring Middleware. As illustrated in the two figures, activation intervals increase on a regular basis. This is because the amount of time needed to occupy the IMMT thread to process data input by channels has increased. For IMMT thread, the activation interval increases every $1000 \mathrm{~ms}$. This corresponds to the interval at which the TMO method used in this experiment transfers data. Fig. 6(b) shows the interval at which the IMMT thread is activated in the Monitoring Middleware. The activation interval pattern is similar to that seen in the Pure Middleware. With the exception of the initial phase where monitoring functions are set, the activation interval varies at $\mu s$ levels. In short, TSDS has little effect on IMMT thread, which operates within the millisecond time frame.

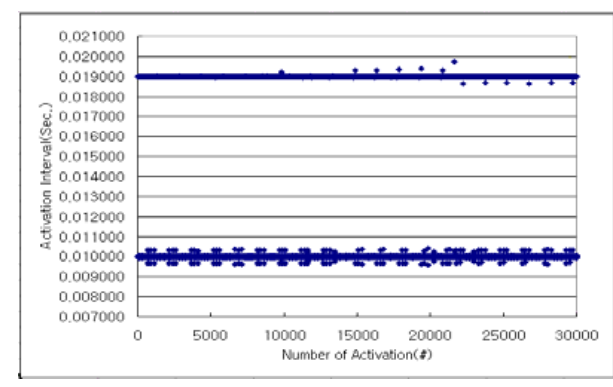

(a) Pure Middleware

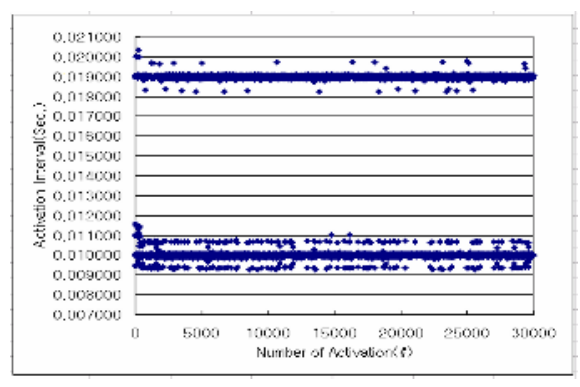

(b) Monitoring Middleware

Fig. 6. A Comparison of Activation Intervals of IMMT

Figs. 7(a) and 7(b) show the interval at which the OMMT thread is activated in the Pure Middleware and Monitoring Middleware. As in IMMT, the amount of time needed to occupy the OMMT thread to process data has increases. In the case of the Monitoring Middleware, as shown in Fig. 7(b), the activation interval pattern is similar to that seen in the Pure Middleware. The OMMT thread in the Monitoring Middleware varies at $\mu s$ levels within $1 \mathrm{~ms}$. In short, TSDS has little effect on OMMT which operates within the millisecond time frame. 


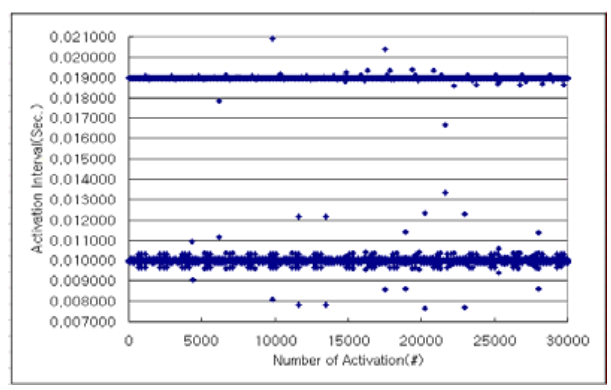

(a) Pure Middleware

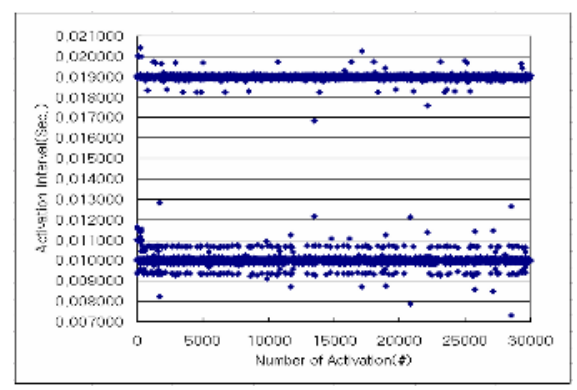

(b) Monitoring Middleware

Fig. 7. A Comparison of Activation Intervals of OMMT

Table 1. Results of t-test by Thread

\begin{tabular}{|c|c|c|c|c|}
\hline \multirow{2}{*}{ Thread } & \multicolumn{4}{|c|}{ t-test } \\
\cline { 4 - 5 } & $\mathrm{t}$ & $\begin{array}{c}\text { significance } \\
\text { probability } \\
\text { Both }\end{array}$ & $\begin{array}{c}\text { An average confidence interval of } 9 \\
9 \%\end{array}$ \\
\cline { 4 - 5 } & & .962 & Lower Limits & Upper Limits \\
\hline IMMT & -.048 & -.00006 & .00006 \\
\hline OMMT & .048 & .962 & -.00006 & .00006 \\
\hline
\end{tabular}

Finally, under the hypothesis that "the Pure Middleware and Monitoring Middleware have similarity in activation intervals," the t-test with the significance level $\alpha=.01$ was conducted to identify the statistical similarity in activation interval among different threads. Table 1 shows the results of analyzing different threads. The significance probability of the IMMT and OMMT is .962. Since .962 > $\alpha=.01$, hypothesis cannot be rejected, as well. This shows that the activation interval of the IMMT and OMMT threads within the two sets of middleware is statistically identical. Overall, the proposed TSDS shows little or no effect on the middleware.

\section{Conclusions and Future Work}

In this paper, TSDS, including both the MODS as a data store for sharing monitoring data among TMO systems and a delivery structure for monitoring data, was proposed. TSDS form a monitoring infrastructure within the middleware, supporting the sharing and delivery of monitoring data for TMO systems. This prevents developer intervention, allowing for automated monitoring functions. The overhead generated by TSDS during experiments are less than $1 \mathrm{~ms}$, having little or no effect on the execution of the middleware. In short, TSDS serves as an infrastructure for providing monitoring functions such as data sharing and data delivery to the middleware, making it ideally suited for monitoring TMO systems. 
Research is currently being conducted to mount TSDS to desktops, embedded systems, and other hardware devices, and is being considered for various system environments in industrial areas such as shipbuilding and power plants.

\section{References}

1. B.A. Schroeder, "On-line Monitoring: A Tutorial," IEEE Computer, 1995

2. B.J. Min, et al., "Implementation of a Run-time Monitor for TMO Programs on Windows NT," IEEE Computer, 2000

3. Hyun-Jun Kim, et al., "TMO-Linux: a Linux-based Real-time Operating System Supporting Execution of TMOs," Proceedings of the 15th IEEE International Symposium on Object-Oriented Real-Time Distributed Computing, 2002

4. J. G Kim, and Cho, S. Y., "LTMOS: An Execution engine for TMO-Based Real-Time Distributed Objects," Proceedings of the PDPTA, 2000

5. Kim, K.H., “APIs for Real-Time Distributed Object Programming," IEEE Computer, 2000

6. Kane Kim, et al., "A Timeliness-Guaranteed Kernel Model: DREAM Kernel and Implementation Techniques," RTCSA, 1995

7. K.H. Kim, and Kopetz, H., "A Real-Time Object Model RTO.k and an Experimental Investigation of Its Potentials," Proceedings of the 18th IEEE Computer Software \& Applications Conference, 1994

8. Yoon-Seok Jeong, Tae Wan Kim, Chun Hyon Chang, "Design and Implementation of a Run-time TMO Monitor on LTMOS," Proceedings of the Embedded Systems and Applications, 2003

9. Yoon-Seok Jeong, Tae Wan Kim, Chun Hyon Chang, "Design of an Architecture for Runtime Process Monitor," Proc. of the 19th KIPS Spring Conference, Vol. 10-1, 2003.

10. S.H. Park, "LTMOS(LinuxTMO System)'s Manual," HUFS, 2000 